Adi abat i c demagnet i zat i on of quadr upol ar nucl ear spi ns in a rotating frame by use of an rf el ectric fiel d

\begin{tabular}{|l|l|}
\hline 著者 & Hat anaka H r oshi , Takahama Nasahi ro \\
\hline $\begin{array}{l}\text { j our nal or } \\
\text { publ i cat i on t i t l e }\end{array}$ & Physi cal Revi ew B \\
\hline vol une & 47 \\
\hline number & 6 \\
\hline page r ange & $3213-3219$ \\
\hline year & 1993 01-01 \\
\hline URL & ht t p: //hdl . handl e. net /2297/7562 \\
\hline
\end{tabular}




\title{
Adiabatic demagnetization of quadrupolar nuclear spins in a rotating frame by use of an rf electric field
}

\author{
Hiroshi Hatanaka and Masahiro Takahama \\ Department of Physics, Faculty of Education, Kanazawa University, Kanazawa 920, Japan
}

(Received 20 July 1992)

\begin{abstract}
Adiabatic demagnetization in a rotating frame (ADRF) has been realized by means of rf-electric-field excitation. The experiments were carried out in the double quantum transition of the ${ }^{27} \mathrm{Al}$ nuclear-spin system in $\mathrm{Al}_{2} \mathrm{O}_{3}$. The excitation was caused by the time-dependent electric quadrupole interaction induced by the rf electric field. Although a strong rf electric field with an intensity of about $14 \mathrm{kV} / \mathrm{cm}$ was applied, the transverse field due to the rf electric field was so small that its role as a heat reservoir could be neglected. The observed ADRF processes and the irreversibility are analyzed in detail with use of Provotorov's saturation theory and by consideration of the spin thermodynamics. The ADRF processes are well described by equations that we have derived from Provotorov's saturation theory. This theoretical treatment is also applicable to the ADRF performed with use of an rf magnetic field under similar experimental conditions.
\end{abstract}

\section{INTRODUCTION}

The concept of a spin temperature in the rotating frame ${ }^{1}$ is very useful for understanding NMR phenomena in solids. In Provotorov's saturation theory, ${ }^{2}$ spin temperatures may be assigned to the Zeeman and the dipolar systems in a rotating frame, and the saturation caused by a resonant $\mathrm{rf}$ magnetic field is described as a thermal mixing between these systems.

Recently, we found ${ }^{3}$ that the concept of spin temperature in a rotating frame is valid when a multilevel spin system is excited by an rf electric field resonant with a double-quantum (DQ) transition (with $\Delta m= \pm 2$ ), and the saturation is described as a thermal mixing in the rotating frame, which is similar to that in the usual NMR setup. The study was performed for ${ }^{27} \mathrm{Al}$ nuclei in $\mathrm{Al}_{2} \mathrm{O}_{3}$. In this case, the saturation is caused by the timedependent electric quadrupole interaction induced by the rf electric field. We refer to the excitation by this interaction as TDEQ excitation.

We also pointed out that the motion of nuclear spins excited by an rf electric field, $E_{0} \cos (\omega t)$, can be described by a vector model similar to that in a conventional NMR setup. We considered a fictitious spin- $-\frac{1}{2}$ system $^{4}$ for the relevant DQ transition between levels $I_{z}=m-1$ and $m+1$ with resonance frequency $\omega_{3}$, and derived an effective Hamiltonian in the rotating frame. This Hamiltonian shows that the spin system is subjected to a longitudinal field $|\delta| / \gamma$ along the $z$ axis and a DQ transverse field $\omega_{p} / \gamma$ in the rotating frame. As a result, the fictitious spins will precess at a frequency $\left(\delta^{2}+\omega_{p}^{2}\right)^{1 / 2}$ around the effective field $\left(\delta^{2}+\omega_{p}^{2}\right)^{1 / 2} / \gamma$ tilted by an angle $\cos ^{-1}\left[\delta /\left(\delta^{2}+\omega_{p}^{2}\right)^{1 / 2}\right]$ from the $z$ axis, where $\delta$ is the frequency offset $\left(\omega_{3}-\omega\right)$ and $\gamma$ is the gyromagnetic ratio of the nuclei. The frequency $\omega_{p}$, which may be called the Rabi frequency in the case of electric-field excitation, is ${ }^{3}$

$\omega_{p}=\left(\omega_{q} / 3 V_{z z}\right) E_{0}\left\langle m+1\left|I_{+}^{2}\right| m-1\right\rangle\left(R_{111}^{2}+R_{222}^{2}\right)^{1 / 2}$,

where $2 \omega_{q}$ is the difference between the transition frequencies of the single quantum (SQ) transitions $m-1$ $\leftrightarrow m$ and $m \leftrightarrow m+1, V_{z z}$ is an element of the intrinsic static electric-field-gradient (EFG) tensor, and $R$ 's are related to the elements of the induced EFG tensor with the electric field, ${ }^{5}$ respectively.

The vector model predicts that several TDEQ analogs of the DQ NMR phenomena, ${ }^{6}$ such as DQ transient nutation, DQ spin echo and DQ spin locking, can be realized in the case of electric-field excitation, if the transverse field $\omega_{p} / \gamma$ is sufficiently large.

In the present paper, we report on the observation of adiabatic demagnetization in a rotating frame (ADRF) realized by means of $\mathrm{rf}$-electric-field excitation in a multilevel system. Two methods of ADRF are usually known; gradually decreasing the frequency offset under the $\mathrm{cw}$ irradiation of an $\mathrm{rf}$ magnetic field, ${ }^{7}$ and gradually decreasing the $\mathrm{rf}$ magnetic field after the spin locking is achieved by a $\frac{1}{2} \pi$ pulse. $^{8}$

We adopted the former method. The frequency offset $\delta$ was gradually reduced to zero under the irradiation of an $\mathrm{rf}$ electric field resonant with the DQ transition. By this procedure, the effective field $\left(\delta^{2}+\omega_{p}^{2}\right)^{1 / 2} / \gamma$ is reduced to $\omega_{p} / \gamma$ with a direction change. If $\omega_{p} / \gamma$ is sufficiently small compared with the local field, the spin system will be demagnetized in the rotating frame. We used a strong rf electric field with an intensity of about 14 $\mathrm{kV} / \mathrm{cm}$, and the magnitude of $\omega_{p} / \gamma$ was found to be negligibly small compared with the local field $H_{L} \cdot\left(\omega_{p} / \gamma\right.$ is about 0.02 times that of $H_{L}$.) Under these experimental conditions, the effective field can be approximated as $|\delta| / \gamma$ even when $|\delta| / \gamma$ becomes smaller than the local field, and the effective field is negligibly small when $|\delta| / \gamma$ becomes comparable to $\omega_{p} / \gamma$.

The ADRF is generally known as a reversible process in magnetic resonance in solids, namely the magnetic order is reversibly transferred between that along the effective field (Zeeman order) and that along the internal dipolar field (dipolar order). However, we found that the 
TDEQ ADRF was considerably irreversible, contrary to expectations. The irreversibility is theoretically analyzed in detail by using Provotorov's saturation theory ${ }^{2,3}$ and spin thermodynamics. Provotorov's saturation theory presents theoretical curves that describe well the ADRF process.

\section{EXPERIMENTAL METHODS AND RESULTS}

The experiments were performed at room temperature on a ${ }^{27} \mathrm{Al}$ nuclear-spin system in a single crystal of $\mathrm{Al}_{2} \mathrm{O}_{3}$ of size $15 \times 10 \times 2 \mathrm{~mm}^{3}$. A static magnetic field $H_{0}$ was applied along the $c$ axis of the crystal to obtain a multilevel system with unequal level spacings and no level mixing. The level system is schematically shown in Fig. 1. We refer to the levels $I_{z}=-3 / 2,-1 / 2$, and $1 / 2$ as 1 , 2 , and 3 , respectively. The strength of $H_{0}$ was adjusted so that the resonance frequency $\omega_{1}$ of the SQ transition $1 \leftrightarrow 2$ was $2 \pi \times 13.00 \mathrm{MHz}$, and $\omega_{3}$ of the DQ transition $1 \leftrightarrow 3$ was $2 \pi \times 25.64 \mathrm{MHz}$. The ADRF was performed for the DQ transition $1 \leftrightarrow 3$.

An $\mathrm{rf}$ electric field with intensity $E_{0}$ of about 14 $\mathrm{kV} / \mathrm{cm}$ was applied, for which $\omega_{p} \simeq 2 \pi \times 0.2 \mathrm{kHz}$. The procedure for the application of a strong rf electric field is almost the same as that in our previous work. ${ }^{3}$ The crystal is processed so that silver electrodes are deposited on the $15 \times 10 \mathrm{~mm}^{2}$ crystal faces parallel to the $c$ axis, and the crystal is covered with epoxy to avoid electric discharge. The sample plays the role of a capacitor of the $L C$ resonance circuit tuned with the frequency of the $\mathrm{rf}$ power $(\simeq 25.64 \mathrm{MHz})$.

For the demagnetization in the rotating frame, we used the sweeping of the frequency $\omega$ of the rf electric field. We have performed two types of experiments with stepwise frequency sweeps $\left(\omega_{3}-\delta_{0}\right) \rightarrow \omega_{3} \rightarrow\left(\omega_{3}-\delta_{0}\right)$ and $\left(\omega_{3}+\delta_{0}\right) \rightarrow \omega_{3} \rightarrow\left(\omega_{3}-\delta_{0}\right)$ as shown below in detail. In both types of experiments, $\delta_{0}=2 \pi \times 18 \mathrm{kHz}$ and the full sweep widths are covered with 16830 steps. In the first type, the full sweep width is $18 \mathrm{kHz}$, and the frequency change by one step is about $1 \mathrm{~Hz}$. On the other hand, those in the second type are $36 \mathrm{kHz}$ and about $2 \mathrm{~Hz}$. These sweep widths are sufficient because the linewidth of

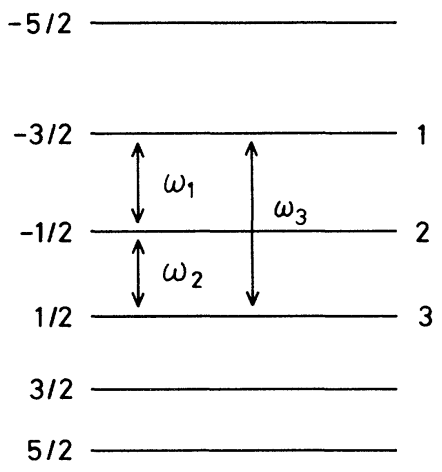

FIG. 1. Energy-level diagram of ${ }^{27} \mathrm{Al}$ nuclei in $\mathrm{Al}_{2} \mathrm{O}_{3}$ subjected to the static magnetic field $H_{0}$ applied along the $c$ axis. No level mixing exists. $\omega_{1}, \omega_{2}$, and $\omega_{3}$ are the transition frequencies between levels 1 and 2, 2 and 3, and 1 and 3, respectively. the DQ transition is about $10 \mathrm{kHz}$ (half-maximum full width $)$ and the transverse field $\omega_{p} / \gamma(\simeq 0.18 \mathrm{Oe})$ is much smaller than the local field $H_{L}=D / \gamma$ of about $8 \mathrm{Oe}$, where $D$ is defined in Sec. III. The stepwise frequency sweep was carried out by changing the voltage of a voltage-controlled oscillator for the rf electric field. These frequency changes by one step are so small that the frequency sweeps can be regarded as continuous and linear.

Since the Zeeman field in the rotating frame is approximated as $|\delta| / \gamma$ even when $|\delta|<H_{L}$, as mentioned in Sec. $I$, the population difference $W$ of the DQ transition represents the degree of the Zeeman order in the rotating frame. However, the sign of $W$ is not necessarily the same as that of the spin temperature of the Zeeman system (Zeeman temperature). For example, when the frequency $\omega$ of the rf electric field is larger than $\omega_{3}$, the positive magnitude of $W$ indicates the Zeeman order at a negative temperature, namely the Zeeman order in the opposite direction to the effective field, because the effective field becomes antiparallel to the $z$ axis (the quantization axis).

The population difference $W$ in the DQ transition cannot be measured directly. In our case $\omega_{3} \simeq 2 \omega_{1}$. Because of this special situation, it can be indirectly measured by doubling the population difference between levels 1 and 2. ${ }^{9}$ However, we adopted a more accurate method using a $\pi$ (rf magnetic) pulse resonant with the $2 \leftrightarrow 3$ transition. The DQ population difference $W$ is converted by this pulse to the SQ one $1 \leftrightarrow 2$, and is measured from the initial amplitude of the free-induction decay (FID) signal at $\omega_{1}$. We call the initial amplitude of this FID signal a Zeeman signal. The $\pi$ pulse can also be used for observing the DQ coherence, which cannot be observed directly. This technique is based on the phenomenon of periodic coherence transfer in a three-level system caused by the resonant SQ transition. ${ }^{10}$

The experimental procedure for the first type of experiment is illustrated in Fig. 2. Just before the rf electric field is turned on at $t=0$, the linear frequency sweep starts. The frequency offset at $t=0$ is $\delta_{0}$. The frequency $\omega$ reaches the resonance point $\omega_{3}$ at $t \simeq 1 \mathrm{~s}$, and the demagnetization process is completed. (The $\mathrm{rf}$ electric field is not zero but it is much smaller than $H_{L}$.) Then it is swept back to the original frequency with the same rate (remagnetization process) in order to examine the reversibility. Trace (a) in Fig. 2 schematically shows the frequency sweep for the demagnetization and the remagnetization processes. The rf electric field is turned off at time $t$ [trace (b)]. Traces (c) and (d) show rf magnetic pulses, a $\pi$ pulse with frequency $\omega_{2}$ followed by a $\frac{1}{2} \pi$ pulse with $\omega_{1}$ [ $\left.\pi\left(\omega_{2}\right)-\frac{1}{2} \pi\left(\omega_{1}\right)\right]$, for the measurement of $W$, where $\omega_{2}$ is the resonance frequency of the 2-3 transition. Repeating the operations (a) to (d) by changing the turn-off time $t$, we obtain time evolutions of $W$ in the demagnetization and remagnetization processes.

We also observed the time evolution of the dipolar order $G$. The dipolar order $G$ is produced from the Zeeman order in the DQ transition. It can be detected in any SQ transition by using a magnetization $\mathrm{rf}$ pulse whose intensity gradually increases 8 [as in trace (e)], or by using a $\frac{1}{4} \pi$ 
pulse $^{11}$ [instead of the pulses $\left.\pi\left(\omega_{2}\right)-\frac{1}{2} \pi\left(\omega_{1}\right)\right]$. We used the former method because of its higher detection efficiency, and measured $G$ from the initial amplitude of the FID signal observed after this rf pulse (dipolar signal).

Open and closed circles in Fig. 3 show the observed time evolutions of $W$ and $G$, respectively. Both the observed Zeeman and dipolar signals are normalized by the Zeeman signal due to the population difference $W_{0}$ in thermal equilibrium with the lattice. The frequency $\omega$ is changed as shown in Fig. $2(\mathrm{a})$, where $\delta_{0}=2 \pi \times 18 \mathrm{kHz}$. As is expected, $W$ gradually decreases to zero together with the growth of $G$. When $W$ becomes zero at $t \simeq 1 \mathrm{~s}$ $\left(\omega=\omega_{3}\right), G$ becomes maximum. These results show that the ADRF is realized when the rf electric field is used instead of the rf magnetic field.

However, it is noted that the Zeeman order lost in the demagnetization process does not completely recover when $\omega$ returns to the original frequency. A considerable degree of order still remains in the dipolar system. In addition, slight kinks showing an irregularity in the ADRF process are recognized in the curves around $t=0.6 \mathrm{~s}$ (shown by arrows).

The ADRF shown in Fig. 3 (the TDEQ ADRF) occurs under positive temperatures, because the direction of the Zeeman field is the same as that of the $z$ axis. The TDEQ ADRF was realized also under negative temperatures by

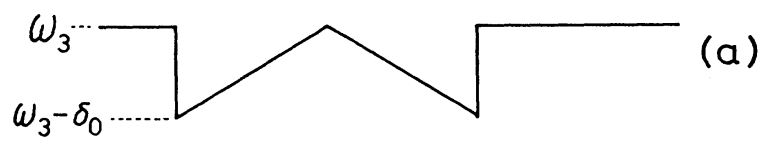

$\omega$

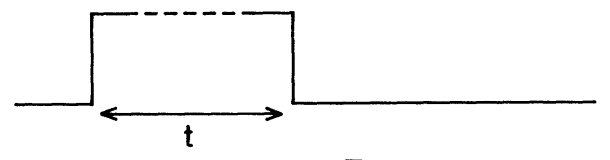

(b)

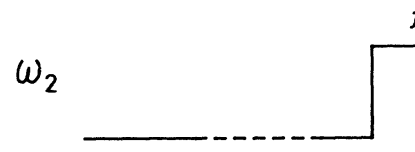

$\pi$

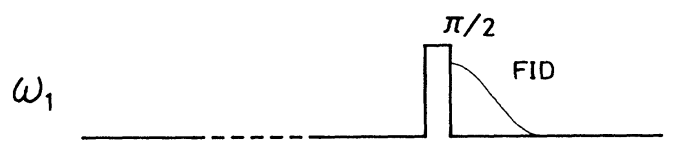

(d)

$\omega_{1}$

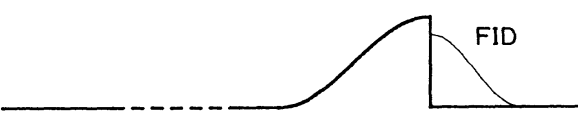

(e)

FIG. 2. Operations of TDEQ ADRF experiments. Just before the $\mathrm{rf}$ electric field is turned on at $t=0$, a linear frequency sweep $\left(\omega_{3}-\delta_{0}\right) \rightarrow \omega_{3} \rightarrow\left(\omega_{3}-\delta_{0}\right)$ starts [trace (a)]. The rf electric field is turned off at time $t$ [trace (b)], and then the Zeeman order $W$ of the DQ transition is measured from the initial amplitude of the FID signal observed by pulses $\pi\left(\omega_{2}\right)-\frac{1}{2} \pi\left(\omega_{1}\right)$ shown by traces (c) and (d). Repetition of these operations with changing the turn-off time $t$ presents the time evolution of $W$ in the process. When the frequency reaches $\omega_{3}$, the rf electric field is not turned off because $\omega_{p} \ll \gamma H_{L}$. In order to observe the time evolution of the dipolar order $G$, the magnetization pulse [trace (e)] is used instead of the pulses $\pi\left(\omega_{2}\right)-\frac{1}{2} \pi\left(\omega_{1}\right)$.

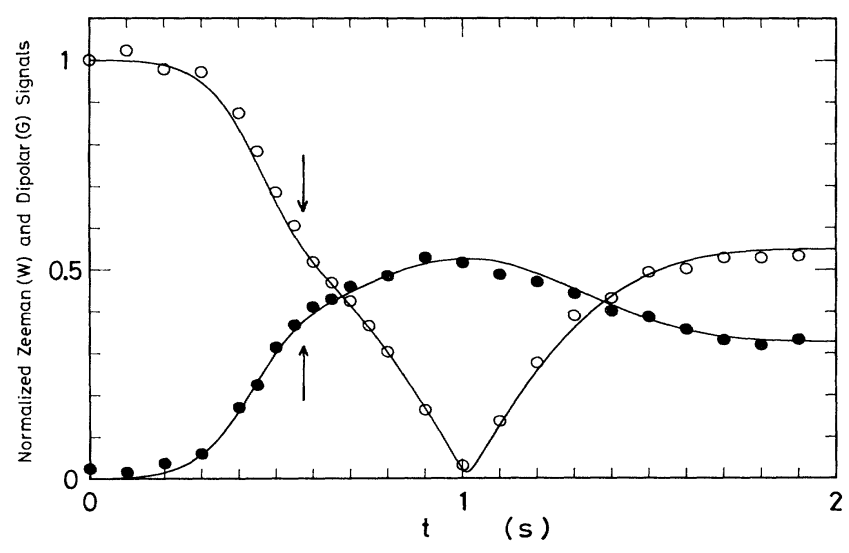

FIG. 3. Processes of the ADRF and of the successive remagnetizations realized in the DQ transition $1 \leftrightarrow 3$ by means of the frequency sweep shown in Fig. 1 with $\delta_{0}=2 \pi \times 18 \mathrm{kHz}$ and $K_{0}=0.0765 \mathrm{~ms}^{-1}$. Open and closed circles show the experimental curves of $W$ and $G$, respectively, and the solid lines are theoretical. $A$ (the fitting factor of the curve of $G$ ) $=0.71$. Arrows indicate the irregularity points of the curves.

sweeping the frequency $\omega$ from $\omega_{3}+\delta_{0}$ to $\omega_{3}$ (instead of $\omega_{3}-\delta_{0}$ to $\left.\omega_{3}\right)$. Open and closed circles in Fig. 4 show the time evolutions of $W$ and $G$ observed by the second type of experiment. (The experimental procedure is the same as that of the first type except for the manner of frequency sweep.) The positive Zeeman signals in the demagnetization process indicate that the Zeeman temperature is negative, because the effective field is antiparallel to the $z$ axis. In the remagnetization process negative Zeeman signals appear. They indicate a negative Zeeman temperature because the effective field is in the direction toward the $z$ axis in this process. The negative dipolar signals in the demagnetization and remagnetization processes are due to the negative dipolar temperature. Therefore, demagnetization and remagnetization occur under negative temperatures. It is also noted that the Zeeman order

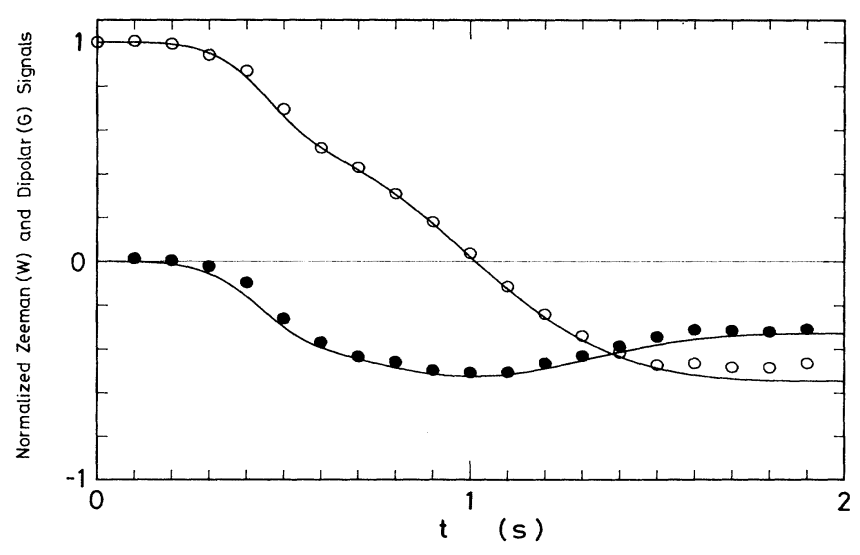

FIG. 4. Similar processes to those in Fig. 3 observed by sweeping the frequency from $\omega_{3}+\delta_{0}$ to $\omega_{3}-\delta_{0}$. The other experimental conditions are the same as those in Fig. 3. Open and closed circles show the experimental curves of $W$ and $G$, respectively, and solid lines are theoretical. $A=0.71$. 
and the dipolar order are not reversibly exchanged as in the case of positive temperatures (Fig. 3).

\section{THEORETICAL ANALYSIS OF THE IRREVERSIBILITY}

As described in Sec. II, the observed ADRF exhibits large irreversibility. In this section, we theoretically analyze the large irreversibility based on Provotorov's saturation theory and on spin thermodynamics.

The observed spin-lattice relaxation times $T_{1}$ of the Zeeman system in the DQ transition and $T_{1 d}$ of the dipolar system were about $18 \mathrm{~s}$ and $30 \mathrm{~s}$, respectively $\left(T_{1 d}\right.$ was longer than $T_{1}$ in this system). These are long enough compared with the full sweep duration of $2 \mathrm{~s}$ of the demagnetization-remagnetization process. Therefore, we do not consider the effect of spin-lattice relaxation. (In the final part of this section, we will take into account the effects of the spin-lattice relaxation.)

We imagine that the linear frequency sweep consists of many small stepwise changes of the frequency $\omega$, that is, the frequency is suddenly shifted by a small amount at $t=n \tau(n=1,2, \ldots)$, where $\tau$ is a short-time interval. The sudden frequency changes produce small temperature differences between the Zeeman and the dipolar systems in the rotating frame. We assume that thermal mixing occurs between the Zeeman system due to $|\delta| / \gamma$, and the dipolar system owing to $\omega_{p}$, and during the intervals, these tend toward a common temperature under a constant frequency offset. The evolutions of $W$ and $G$ during each interval can be described by use of Provotorov's saturation theory for rf-electric-field excitation. ${ }^{3}$ The amounts of $W$ and $G$ at the end of the $n$th interval are those at the beginning of the $(n+1)$ th interval, because the change in the frequency offset from $\delta_{n}$ (at interval $n$ ) to $\delta_{n+1}$ itself causes no changes in the amounts of $W$ and $G$. Since $\tau$ is much shorter than the sweep duration, the initial amounts $W_{n}$ and $G_{n}$ at the interval $n$ can be regarded as representative ones. Thus, we obtain the following equations from Eqs. (3) in Ref. 3:

$$
\begin{aligned}
& W_{n+1}=B_{n}\left(D^{2} W_{n}-D \delta_{n} G_{n}\right)+W_{n}, \\
& G_{n+1}=B_{n}\left(\delta_{n}^{2} G_{n}-D \delta_{n} W_{n}\right)+G_{n},
\end{aligned}
$$

with

$$
\begin{aligned}
& D=\left[\operatorname{Tr}\left(\mathcal{H}_{d}^{* 2} / \operatorname{Tr}\left(S_{z}^{2}\right)\right]^{1 / 2},\right. \\
& B_{n}=\left\{\exp \left[-K_{n}\left(1+\delta_{n}^{2} / D^{2}\right) \tau\right]-1\right\} /\left(D^{2}+\delta_{n}^{2}\right),
\end{aligned}
$$

where $\mathscr{H}_{d}^{*}$ is the truncated dipolar interaction Hamiltonian for the multilevel system, ${ }^{12} S_{z}$ is the $z$ component of the fictitious spin- $\frac{1}{2}$ operator ${ }^{4}$ for the DQ transition, and $K_{n}$ is the saturation rate at $\delta_{n} . K_{n}$ is given by assuming a Gaussian line shape with a second moment $T_{2}^{-2}$ as

$$
K_{n}=K_{0} \exp \left(-\frac{1}{2} \delta_{n}^{2} T_{2}^{2}\right),
$$

where

$$
K_{0}=\frac{1}{2} \sqrt{2 \pi} \omega_{p}^{2} T_{2}
$$

is the saturation rate at exact resonance. The value of $B_{n}$ in Eqs. (3) is approximated as $-K_{n} \tau / D^{2}$ since $\tau$ is very short.

Solid lines in Figs. 3 and 4 show the calculated values of $W_{n}$ and $G_{n}$ from Eqs. (2), where we use $K_{0}=0.0765$ $\mathrm{ms}^{-1}$ obtained from our saturation experiment at exact resonance, ${ }^{3}$ and $D=2 \pi \times 8.86 \mathrm{kHz}^{13}$ and $T_{2}=38 \mu \mathrm{s}^{12}$ Values of $W_{n}$ and $G_{n}$ are plotted in units of $W_{0}$. The intensity of the observed dipolar signal does not correctly give the degree of the dipolar order, because of the magnetization loss due to the incompleteness of the magnetization pulse [trace (e)]. We plot the values of $A G_{n}$ with a fitting factor $A=0.71$. These curves are in excellent agreement with experimental curves. Patterns of the curves for a constant duration of the sweep do not essentially depend on the number of the frequency steps, provided that the number is large. Nearly the same patterns as those in Figs. 3 and 4 were obtained experimentally and theoretically for the frequency sweep of only 130 steps. Therefore, the curves in Figs. 3 and 4 can be regarded as those for a continuous sweep. Since the experimental results can be explained by Eqs. (2) without any use of the spin-lattice relaxation terms, we can conclude that the observed ADRF processes are adiabatic.

Therefore, the irreversibility of the process should be attributed to the internal thermal mixing of the system. In order to examine the effect of the thermal mixing, we draw theoretical curves when the change occurs very slowly, or the sweep duration is very long. Solid lines in Fig. 5 are the theoretical curves of the time evolutions of $W$ and $G$ for the sweep duration of $20 \mathrm{~s}$ and the sweep width of $20 \mathrm{kHz}$. (Other conditions are the same as those in Fig. 3.) These are ideal curves obtained if the spinlattice relaxation times are infinite. Although the reversibility is improved, a large irreversibility still exists. A similar theoretical result was also obtained by increasing $\omega_{p}$.

It is noted that there are kinks of the curves at $t \sim 4 \mathrm{~s}$. We call them irregularity points. It is also noted that the recovered Zeeman order and the remaining dipolar order

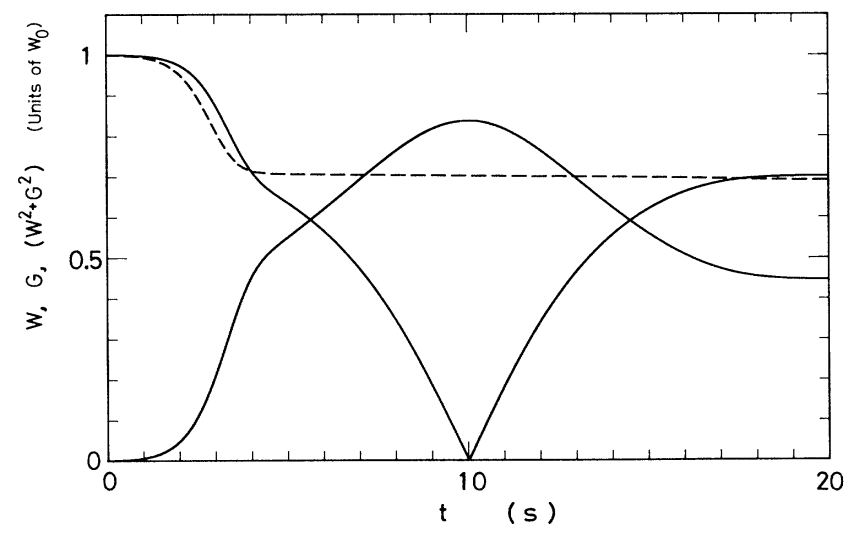

FIG. 5. Time evolutions of $W$ and $G$ in the adiabatic processes theoretically obtained with the sweep duration of $20 \mathrm{~s}$ (solid lines). $K_{0}=0.0765 \mathrm{~ms}^{-1}$ and $\delta_{0}=2 \pi \times 20 \mathrm{kHz}$. The dashed line is the time evolution of $W^{2}+G^{2}$, showing that the entropy suddenly increases in the early process of the demagnetization, and after that, the increase becomes very small. 
at the end of the sweep are almost equal to the orders around the irregularity points. It shows that the ADRF process is quite irreversible before the irregularity points, but is almost reversible beyond these points.

We will confirm the features described above by evaluating the entropy of the spin system. Since it is not ascertained that the spin system is always in thermal equilibrium in the rotating frame during the frequency sweep, we use the density matrix $\rho$ in the rotating frame with two different temperatures (the Zeeman temperature $T_{z}$ and the dipolar one $T_{d}$ ) as

$$
\rho=\exp \left(\hbar \delta S_{z} / k T_{z}-\hbar \mathcal{H}_{d}^{*} / k T_{d}\right) / Z,
$$

where $k$ is the Boltzmann constant and

$$
Z=\operatorname{Tr}\left[\exp \left(\hbar \delta S_{z} / k T_{z}-\hbar \mathcal{H}_{d}^{*} / k T_{d}\right)\right] .
$$

The notation $\delta, W, G$, etc, without the subscript $n$ denotes continuous functions of the time $t$. Philippot shows that the introduction of the different temperatures is valid if the high-temperature approximation holds. ${ }^{14}$

Therefore, the entropy $S=-k \operatorname{Tr}(\rho \ln \rho)$ is represented, in high-temperature approximation, as

$$
S=-\frac{1}{2} C \delta^{2} / T_{z}^{2}-\frac{1}{2} C D^{2} / T_{d}^{2}+k N \ln (2 I+1),
$$

where $N$ is the number of spins and $C=\hbar^{2} \operatorname{Tr}\left(S_{x}^{2}\right) / k \operatorname{Tr}(1)$ is the nuclear Curie constant. Since the population difference and the dipolar order are given by ${ }^{3}$

$$
\begin{aligned}
& W=2 \operatorname{Tr}\left(S_{z} \rho\right)=2 C \delta / \hbar T_{z}, \\
& G=2 C D / \hbar T_{d},
\end{aligned}
$$

the entropy is obtained as

$$
S=-\hbar^{2}\left(W^{2}+G^{2}\right) / 8 C+k N \ln (2 I+1) .
$$

The dashed line in Fig. 5 shows the values $W_{n}^{2}+G_{n}^{2}$. It indicates that the entropy increases suddenly before the irregularity points, and is almost constant thereafter.

The large entropy change is interpreted as follows: even if the frequency is swept slowly, the thermal mixing does not occur in the rotating frame until the frequency $\omega$ reaches the edge of the resonance line. When the frequency reaches the edge, the Zeeman system is cooled to $T_{z}=T_{L} \delta^{\prime} / \omega_{3}$ in the rotating frame, whereas $T_{d}$ is still the lattice temperature $T_{L}$, where $\delta^{\prime}$ is the frequency offset at the edge. The resultant large temperature difference produces the large increase in the entropy. This is the behavior observed before the irregularity points. Goldman et al. pointed out this effect in the study of adiabatic fast passage by the rf magnetic field on ${ }^{19} \mathrm{~F}$ nuclei in $\mathrm{CaF}_{2} \cdot{ }^{15}$ However, their approximation of the sudden establishment of equilibrium at the edge of the line is not valid in this case, and the irreversible loss of Zeeman order observed in this work was much larger than that estimated by them on ${ }^{19} \mathrm{~F}$ nuclei.

After the irregularity points, the process can be regarded as an isoentropic one, indicating that the temperature difference becomes very small. It is known that the change in the magnetization in the ideal adiabatic demagnetization in the rotating frame or in the laboratory frame is described by an equation ${ }^{7}$ which is derived by taking the entropy of the system characterized by a single temperature to be constant. We examine whether or not the curve of $W$ after the irregularity points in Fig. 5 is described by such an equation. Since the change in $\delta$ itself does not directly cause the changes in $W$ and $G$ as mentioned above, $d W / d t$ and $d G / d t$ are produced only by the thermal mixing during each interval. If the changes of $W, G$, and $\delta$ between adjacent frequency steps are sufficiently small, we can regard them as continuous changes. Then, $\left(W_{n+1}-W_{n}\right) / \tau$ and $\left(G_{n+1}-G_{n}\right) / \tau$ can be replaced by $d W / d t$ and $d G / d t$, respectively, and the following equations are obtained from Eqs. (2) with the aid of Eqs. (9):

$$
\begin{aligned}
& d W / d t=-2 C \delta K\left(1 / T_{z}-1 / T_{d}\right) / \hbar, \\
& d G / d t=-2 C \delta^{2} K\left(1 / T_{d}-1 / T_{z}\right) / \hbar D .
\end{aligned}
$$

The rate of the entropy increase is given by

$$
d S / d t=-\hbar^{2}(W d W / d t+G d G / d t) / 4 C .
$$

Substituting Eqs. (9) and (11) into Eq. (12), we obtain

$$
d S / d t=C K \delta^{2}\left(1 / T_{z}-1 / T_{d}\right)^{2} .
$$

Equations (11) and (13) indicate that the decrease of the temperature difference [more precisely, the difference between the reciprocal temperatures $\left.\left(1 / T_{z}-1 / T_{d}\right)\right]$ does not only suppress the increase in the entropy, but also decreases the rate of transfer of the order from the Zeeman system to the dipolar one. However, the rate of the entropy increase depends on the square of the reciprocaltemperature difference, while $d W / d t$ and $d G / d t$ depend on the difference itself. Therefore, the rate of the entropy increase decreases dominantly as the temperature difference decreases.

Thus, we can consider the isoentropic ADRF process to be realized, if the reciprocal-temperature difference becomes so small that its square may be approximated as zero. The process after the irregularity points shown in Fig. 5 is considered to be in this situation. To this approximation, the entropy generally given by Eq. (8) is considered to be constant during the process. Letting the right-hand side of Eq. (8) be constant, we obtain the following equation using Eqs. (9):

$$
W\left(\delta^{2}+D^{2}\right)^{1 / 2} / \delta=\text { const },
$$

which is essentially the same as the above-mentioned equation ${ }^{7}$ for ideal adiabatic demagnetization and remagnetization processes. In the derivation of Eq. (14), the term including the reciprocal-temperature difference itself is neglected because it is very small. Replacing $\delta$ by an appropriate linear function of $t$, we recognize that Eq. (14) describes well the $W$ curve after the irregularity points in Fig. 5. There is a slight discrepancy between the tail ends of these remagnetization curves. This discrepancy is explained by the fact that the thermal mixing becomes less sufficient as the frequency $\omega$ moves far away from $\omega_{3}$, where this effect is not included in Eq. (14).

The experimental results in Figs. 3 and 4 also show the above features of entropy evolution. The entropy sud- 
denly increases in the early stage of the process (before the irregularity point). After about $t=0.6 \mathrm{~s}$ the entropy increase becomes very small, though it is slightly larger than that shown in Fig. 5. Thus we can conclude that the reversible ADRF can occur also by TDEQ excitation due to an rf electric field, if we start with $T_{z}=T_{d}$ in the rotating frame.

We finally consider the effects of the spin-lattice relaxation on the TDEQ ADRF. In view of the good agreement between the theoretical and the experimental curves of the recovering Zeeman order, as shown in Fig. 3, we cannot neglect the disagreement in the recovery process of $W$ in Fig. 4. The disagreement became large when the sweep duration was lengthened. We expect the slight disagreement to be due to effects of the spin-lattice relaxation in the experimental curve.

If we can assume that the variation rates $\left(W_{n+1}\right.$ $\left.-W_{n}\right) / \tau$ and $\left(G_{n+1}-G_{n}\right) / \tau$ are given by superpositions of the effects of the thermal mixing and of the spin-lattice relaxations, Eqs. (2) are modified as

$$
\begin{aligned}
& W_{n+1}=B_{n}\left(D^{2} W_{n}-D \delta_{n} G_{n}\right)+W_{n}-\left(W_{n}-W_{0}\right) \tau / T_{1}, \\
& G_{n+1}=B_{n}\left(\delta_{n}^{2} G_{n}-D \delta_{n} W_{n}\right)+G_{n}-G_{n} \tau / T_{1 d} .
\end{aligned}
$$

Solid lines in Fig. 6 are the theoretical curves plotted by using Eqs. (15) with the experimental values of $T_{1}=18$ $\mathrm{s}$ and $T_{1 d}=30 \mathrm{~s}$. The agreement between the theoretical curves and the experimental ones [shown by open $(W)$ and closed $(G)$ circles] is very good, for a full sweep duration of $3 \mathrm{~s}$. The dashed lines are the theoretical curves due to Eqs. (2). (The patterns of the solid and the dashed lines coincide in the demagnetization process.) In the case of the recovery process shown in Fig. 3, the theoretical curves due to Eqs. (15) were almost the same as those due to Eqs. (2).

\section{CONCLUDING REMARKS}

The ADRF was realized by use of an rf electric field in the DQ transition of the ${ }^{27} \mathrm{Al}$ nuclear-spin system in $\mathrm{Al}_{2} \mathrm{O}_{3}$ at room temperature. The transverse field due to the TDEQ interaction induced by the rf electric field is so small that its role as a heat reservoir can be neglected. The observed TDEQ ADRF process is well described by equations derived from Provotorov's saturation theory for TDEQ excitation. Introducing the effects of the spin-lattice relaxations in the Zeeman and the dipolar systems into the equations, we obtain more precise theoretical curves. From theoretical studies based on Provotorov's saturation theory and on spin thermo-

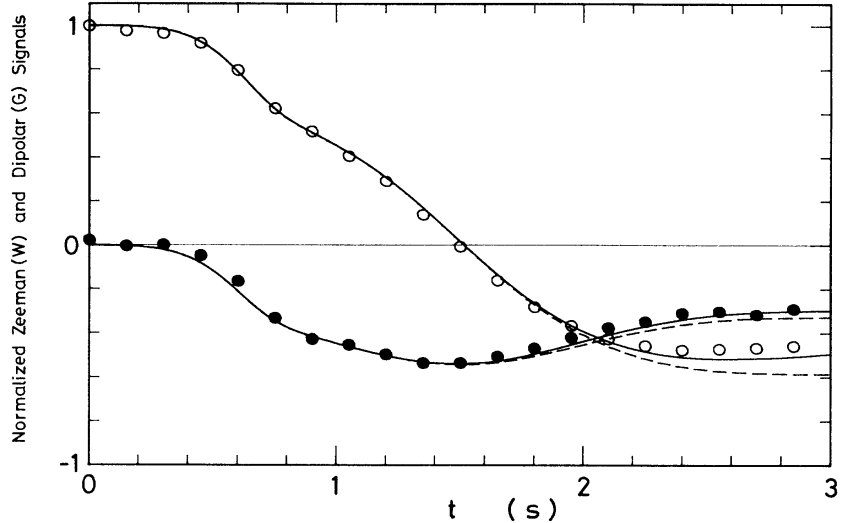

FIG. 6. Corrections of the theoretical curves of the adiabatic process made by taking into account the effects of the spinlattice relaxations with $T_{1}=18 \mathrm{~s}$ and $T_{1 d}=30 \mathrm{~s}$ (solid lines). The experimental conditions are the same as those in Fig. 4 except for the sweep duration (3s). Dashed lines are the theoretical curves due to Eqs. (2).

dynamics, it is concluded that reversible ADRF due to TDEQ excitation is possible.

However, the large irreversible loss of the Zeeman order was observed in the early stage of the ADRF process. This irreversible loss it due to the large temperature difference between the Zeeman and the dipolar systems produced when the frequency $\omega$ reaches the edge of the line. This type of irreversible loss is also produced in ADRF with use of rf-magnetic-field excitation. We examined experimentally and theoretically the reversibilities for the DQ and SQ excitations of this spin system, and for the excitation of the ${ }^{19} \mathrm{~F}$ spin system in $\mathrm{CaF}_{2}$. We obtained the result that the irreversible loss is large for DQ and SQ excitations, but not for the ${ }^{19} \mathrm{~F}$ spin system as pointed out by Goldman et al. Thus, the large irreversible loss is not due to characteristics of TDEQ excitation but to that of the multilevel system. The study of ADRF's performed by means of rf-magnetic-field excitation in those spin systems will be published elsewhere.

\section{ACKNOWLEDGMENTS}

We are grateful to Professor T. Hashi for his valuable comments. We would like to thank Mr. H. Arakawa of Yokaichi Plant, Murata Mfg. Co., Ltd., for his assistance in sample processing with epoxy powder. This work is supported by a Grant-in-Aid for Scientific Research from the Ministry of Education, Science and Culture.
${ }^{1}$ A. G. Redfield, Phys. Rev. 98, 1787 (1955).

${ }^{2}$ B. N. Provotorov, Zh. Eksp. Teor. Fiz. 41, 1582 (1961) [Sov. Phys. JETP 14, 1126 (1962)]; M. Goldman, Spin Temperature and Nuclear Magnetic Resonance in Solids (Oxford University, New York, 1970), p. 75.

${ }^{3}$ H. Hatanaka, Phys. Rev. B 40, 289 (1989).

${ }^{4}$ A. Abragam, The Principles of Nuclear Magnetism (Oxford University, New York, 1961); H. Hatanaka, H. Deguchi, and
T. Hashi, J. Phys. Soc. Jpn. 54, 374 (1985).

${ }^{5}$ R. W. Dixon and N. Bloembergen, Phys. Rev. 135, A1669 (1964).

${ }^{6}$ H. Hatanaka and T. Hashi, J. Phys. Soc. Jpn. 39, 1139 (1975); S. Vega and A. Pines (unpublished); H. Hatanaka and T. Hashi, Phys. Lett. 67A, 183 (1978); H. Hatanaka and T. Hashi, Phys. Rev. B 27, 4095 (1983), and references therein. ${ }^{7}$ C. P. Slichter and W. C. Holton, Phys. Rev. 122, 1701 (1961). 
${ }^{8}$ A. G. Anderson and S. R. Hartmann, Phys. Rev. 128, 2023 (1962).

${ }^{9}$ H. Hatanaka and N. Tabuchi, J. Phys. Soc. Jpn. 56, 1323 (1987).

${ }^{10}$ H. Hatanaka, T. Terao, and T. Hashi, J. Phys. Soc. Jpn. 39 835 (1975).

11J. Jeener and P. Broekaert, Phys. Rev. 157, 232 (1967).
${ }^{12}$ H. Hatanaka and T. Hashi, J. Phys. Soc. Jpn. 50, 3629 (1981).

${ }^{13}$ The value of $D$ in this paper is different from that quoted in Ref. 3, because of a slight difference in definition.

${ }^{14}$ J. Philippot, Phys. Rev. 133, A471 (1964).

${ }^{15}$ M. Goldman, M. Chapellier, and V. H. Chau, Phys. Rev. 168, 301 (1968). 